\title{
11 Textiles and other manufactures
}

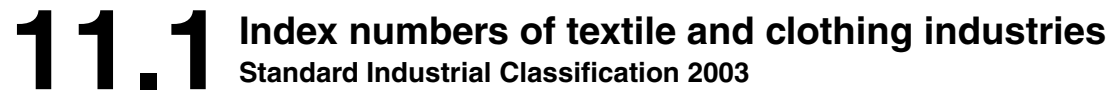

$2003=100$, seasonally adjusted

\begin{tabular}{|c|c|c|c|c|}
\hline & Manufacture of textiles & $\begin{array}{l}\text { Manufacture of textile fibres, } \\
\text { weaving \& finishing of textiles }\end{array}$ & $\begin{array}{r}\text { Manufacture of textile articles } \\
\text { (exc. apparel), } \\
\text { knitted/crocheted goods }\end{array}$ & $\begin{array}{l}\text { Manufacture of clothing } \\
\text { including dressing fur }\end{array}$ \\
\hline \multicolumn{5}{|c|}{$\begin{array}{l}\text { Based on SIC } 2003 \\
\text { classification }\end{array}$} \\
\hline & 17 & $17.1-3$ & $17.4-7$ & 18 \\
\hline $\begin{array}{l}2005 \\
2006 \\
2007 \\
2008\end{array}$ & $\begin{array}{l}\text { AIMS } \\
90.2 \\
88.9 \\
85.9 \\
85.5\end{array}$ & $\begin{array}{r}\text { H4PA } \\
82.1 \\
70.6 \\
66.2 \\
63.2\end{array}$ & $\begin{array}{r}\text { H4PB } \\
94.0 \\
97.5 \\
95.2 \\
96.0\end{array}$ & $\begin{array}{r}\text { AIMT } \\
89.6 \\
91.8 \\
89.9 \\
92.8\end{array}$ \\
\hline $\begin{array}{r}2005 \text { Q3 } \\
\text { Q4 }\end{array}$ & $\begin{array}{l}89.3 \\
89.2\end{array}$ & $\begin{array}{l}81.6 \\
78.0\end{array}$ & $\begin{array}{l}92.9 \\
94.5\end{array}$ & $\begin{array}{l}90.0 \\
88.2\end{array}$ \\
\hline $\begin{array}{r}2006 \text { Q1 } \\
\text { Q2 } \\
\text { Q3 } \\
\text { Q4 }\end{array}$ & $\begin{array}{l}90.6 \\
89.8 \\
87.8 \\
87.4\end{array}$ & $\begin{array}{l}73.4 \\
71.4 \\
69.7 \\
68.1\end{array}$ & $\begin{array}{l}98.7 \\
98.5 \\
96.3 \\
96.5\end{array}$ & $\begin{array}{l}93.2 \\
89.5 \\
90.1 \\
94.5\end{array}$ \\
\hline $\begin{array}{r}2007 \text { Q1 } \\
\text { Q2 } \\
\text { Q3 } \\
\text { Q4 }\end{array}$ & $\begin{array}{l}85.6 \\
86.1 \\
86.2 \\
85.9\end{array}$ & $\begin{array}{l}66.5 \\
66.8 \\
67.6 \\
64.0\end{array}$ & $\begin{array}{l}94.6 \\
95.1 \\
94.9 \\
96.2\end{array}$ & $\begin{array}{l}85.2 \\
91.8 \\
92.3 \\
90.4\end{array}$ \\
\hline $\begin{array}{r}2008 \text { Q1 } \\
\text { Q2 } \\
\text { Q3 } \\
\text { Q4 }\end{array}$ & $\begin{array}{l}86.7 \\
87.0 \\
86.2 \\
82.1\end{array}$ & $\begin{array}{l}68.1 \\
65.2 \\
61.1 \\
58.5\end{array}$ & $\begin{array}{l}95.5 \\
97.3 \\
98.0 \\
93.2\end{array}$ & $\begin{array}{l}90.1 \\
90.1 \\
94.2 \\
96.9\end{array}$ \\
\hline 2009 Q1 & 80.9 & 55.4 & 92.9 & 86.9 \\
\hline $\begin{array}{c}2008 \text { Mar } \\
\text { Apr } \\
\text { May } \\
\text { Jun }\end{array}$ & $\begin{array}{l}86.8 \\
87.7 \\
86.7 \\
86.6\end{array}$ & $\begin{array}{l}67.1 \\
67.5 \\
64.4 \\
63.6\end{array}$ & $\begin{array}{l}96.0 \\
97.2 \\
97.2 \\
97.4\end{array}$ & $\begin{array}{l}91.2 \\
89.6 \\
90.4 \\
90.5\end{array}$ \\
\hline $\begin{array}{l}\text { Jul } \\
\text { Aug } \\
\text { Sep } \\
\text { Oct } \\
\text { Nov } \\
\text { Dec }\end{array}$ & $\begin{array}{l}88.1 \\
85.8 \\
84.6 \\
83.3 \\
82.1 \\
81.1\end{array}$ & $\begin{array}{l}62.3 \\
60.8 \\
60.2 \\
59.8 \\
59.0 \\
56.8\end{array}$ & $\begin{array}{r}100.3 \\
97.5 \\
96.1 \\
94.4 \\
92.9 \\
92.5\end{array}$ & $\begin{array}{r}93.3 \\
95.2 \\
94.1 \\
92.5 \\
100.7 \\
97.6\end{array}$ \\
\hline $\begin{array}{r}2009 \text { Jan } \\
\text { Feb } \\
\text { Mar }\end{array}$ & $\begin{array}{l}80.5^{\dagger} \\
81.9 \\
80.3\end{array}$ & $\begin{array}{l}56.4^{\dagger} \\
55.4 \\
54.3\end{array}$ & $\begin{array}{l}91.8^{\dagger} \\
94.3 \\
92.6\end{array}$ & $\begin{array}{l}90.1^{\dagger} \\
86.9^{-9} \\
83.7^{-1}\end{array}$ \\
\hline
\end{tabular}

Note: ONS has introduced metholological changes to the Index of Production from March 2008 (January 2008 data). Also, a new industry structure Source: Office for National Statistics : 01633456492 means that the level of detail published is reduced. For the textile and clothing sector, industries are no longer available at the full level of disaggregation. Further information can be found on the NS website at http://www.statistics.gov.uk/cci/article.asp?id=1908. 
Total UK manufacturers' sales by industry

$£$ Thousand

\begin{tabular}{|c|c|c|c|c|c|c|}
\hline & \multicolumn{3}{|c|}{ Household textiles } & \multirow{2}{*}{$\begin{array}{r}\text { Non-woven excluding } \\
\text { apparel }\end{array}$} & \multirow{2}{*}{$\begin{array}{r}\text { Canvas goods, sacks } \\
\text { etc }\end{array}$} & \multirow{2}{*}{$\begin{array}{r}\text { Cordage rope, twine \& } \\
\text { netting }\end{array}$} \\
\hline & Soft furnishings & Household textiles & Carpets \& rugs & & & \\
\hline Subclass (SIC 2003) & 17401 & 17403 & 17510 & 17530 & 17402 & 17520 \\
\hline & CKPE & CKPF & CKPG & $\mathrm{CKPH}$ & CKPI & CKPJ \\
\hline 1995 & 384444 & 887255 & 1095811 & 138380 & 159022 & 80079 \\
\hline 1996 & 450797 & 988302 & 1117321 & & 167491 & 79730 \\
\hline 1997 & 466574 & 902675 & 1053675 & 135127 & 150931 & \\
\hline 1998 & 493515 & 914508 & 1083142 & 144617 & 127373 & 82656 \\
\hline 1999 & 443718 & 941637 & 1028911 & 157963 & 161172 & 80103 \\
\hline 2000 & 458407 & 986779 & 972988 & 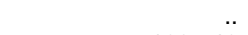 & 136549 & 78587 \\
\hline 2001 & 544307 & 896819 & 902029 & 168546 & 137455 & 101626 \\
\hline 2002 & 528697 & 780557 & 840517 & 167013 & 116602 & 86281 \\
\hline 2003 & 592336 & 730484 & 750565 & 152734 & 112634 & 87307 \\
\hline 2004 & 575924 & 653817 & 689946 & 149188 & 100897 & 75531 \\
\hline 2005 & 564267 & 643337 & 711459 & 150282 & 80457 & 83972 \\
\hline 2007 & 646307 & 686072 & 772757 & 169164 & .. & 85378 \\
\hline
\end{tabular}

\begin{tabular}{|c|c|c|c|c|c|}
\hline & \multicolumn{3}{|c|}{ Knitted and crocheted } & \multirow[b]{2}{*}{ Lace $^{1}$} & \multirow[b]{2}{*}{ Narrow fabrics ${ }^{2}$} \\
\hline & Fabrics & Hosiery & $\begin{array}{r}\text { Pullovers, cardigans \& } \\
\text { similar articles }\end{array}$ & & \\
\hline Subclass (SIC 2003) & 17600 & 17710 & 17720 & 17541 & 17542 \\
\hline & CKPK & CKPL & CKPM & CKPN & CKPO \\
\hline 1995 & 605671 & 521920 & 920516 & .. & 281000 \\
\hline 1997 & 600559 & 525352 & 960748 & .. & 255000 \\
\hline 1998 &.. & 451270 & 771029 & 62000 & 236000 \\
\hline 1999 &.. & 363046 & 663533 & 39000 & 223000 \\
\hline 2000 & 374153 & & 581936 & 34000 & 210000 \\
\hline 2001 & & 282195 & 406567 & 28000 & 193000 \\
\hline 2002 & 244325 & & 351497 & 23442 & 186973 \\
\hline 2003 & 202658 & 241318 & 306854 & 17874 & 159949 \\
\hline 2004 & 197031 & .. & 219492 & 15709 & 144529 \\
\hline 2006 & .. & .. & 175627 & 15379 & 123511 \\
\hline 2007 &.. & .. & 155173 &.. & 110316 \\
\hline
\end{tabular}

1 Data prior to 2002 are estimates to two digits

2 Data prior to 2002 are estimates to three digits 
Wearing apparel, dressing and dyeing of fur, leather clothes

Total UK manufacturers' sales by industry

$£$ Thousand

\begin{tabular}{|c|c|c|c|c|c|c|c|c|c|}
\hline & \multirow[b]{2}{*}{ Workwear } & \multicolumn{2}{|c|}{ Outerwear } & \multicolumn{2}{|c|}{ Underwear } & \multirow[b]{2}{*}{ Hats } & \multirow{2}{*}{$\begin{array}{r}\text { Other \& } \\
\text { accessories }\end{array}$} & \multirow{2}{*}{$\begin{array}{r}\text { Dressing \& } \\
\text { dyeing of fur \& } \\
\text { articles of fur }\end{array}$} & \multirow[b]{2}{*}{ Leather clothes } \\
\hline & & Men's & Women's & Men's & Women's & & & & \\
\hline & CKPP & CKPQ & CKPR & CKPS & CKPT & CKPU & CKPV & CKPW & CKPX \\
\hline 1995 & 243104 & 1011014 & 1732424 & 499251 & 1044030 & 86083 & 680616 & 25285 & 25285 \\
\hline 1996 & 261516 & 1020817 & 1603330 & 545995 & 1013685 & 93621 & 737665 & 24711 & .. \\
\hline 1997 & 292143 & 905613 & 1662377 & 559624 & 1018358 & 89752 & 681916 & & \\
\hline 1998 & 290200 & 759379 & 1302131 & 572157 & 938045 & 70986 & 661375 & 6635 & 19786 \\
\hline 1999 & 278733 & 612538 & 1001239 & 436778 & 779013 & 62653 & 603545 & 5763 & 13941 \\
\hline 2000 & 260798 & 421406 & 915310 & 293713 & 736768 & $\cdot$ & 568286 & 4541 & 15580 \\
\hline 2001 & 243942 & 329441 & 789034 & 230017 & 612113 & .. & 458405 & 6512 & 13255 \\
\hline 2002 & 270889 & 283160 & 876338 & 220014 & 552990 & 45156 & 420323 & 6297 & 10529 \\
\hline 2003 & 287388 & 291866 & 709078 & 195039 & 457567 & 37115 & 361555 & 3922 & 8534 \\
\hline 2004 & 262704 & 248638 & 791793 & 171318 & 392406 & 34712 & 314666 & 4467 & 6678 \\
\hline 2005 & 224642 & 181704 & 632078 & & 349589 & & 280000 & 4143 & 5198 \\
\hline 2007 & 198353 & 167188 & 540932 & 63205 & .. & 28424 & 265162 & 2587 & 4258 \\
\hline
\end{tabular}

Miscellaneous products - goods not classified elsewhere
Total UK manufacturers' sales by industry

$£$ Thousand

\begin{tabular}{|c|c|c|c|}
\hline & Pumps & Compressors & Taps \& valves \\
\hline \multirow[t]{2}{*}{ Subclass (SIC 2003) } & 29121 & 29122 & 29130 \\
\hline & CKPY & CKPZ & CKQA \\
\hline 1995 & 926666 & 1067179 & 1194584 \\
\hline 1996 & 1003912 & 1082552 & 1384408 \\
\hline 1997 & 1090032 & 1085939 & 1330475 \\
\hline 1998 & 1023674 & 1223879 & 1377470 \\
\hline 2000 & 1031341 & 1352122 & 1192583 \\
\hline 2001 & 989670 & 1236154 & 1162459 \\
\hline 2002 & 1021177 & 1087306 & 1115055 \\
\hline 2003 & 1132880 & 1079040 & 1103264 \\
\hline 2004 & 1156732 & 1176542 & 1164350 \\
\hline 2005 & 1220381 & 1085746 & 1237324 \\
\hline 2006 & 1233254 & 1239712 & 1269060 \\
\hline
\end{tabular}

